\title{
Nonhydrogenic exciton spectrum in perovskite $\mathrm{CH}_{3} \mathrm{NH}_{3} \mathrm{Pbl}_{3}$
}

\author{
E. Menéndez-Proupin ，Carlos L. Beltrán Ríos , and P. Wahnón
}

Keywords metal-halide perovskites, polarons, dielectric properties, excitons, $\mathrm{CH}_{3} \mathrm{NH}_{3} \mathrm{PbI}_{3}$

The excitons in the orthorhombic phase of the perovskite $\mathrm{CH}_{3} \mathrm{NH}_{3} \mathrm{PbI}_{3}$ are studied using the effective mass approximation. The electron-hole interaction is screened by a distancedependent dielectric function, as described by the Haken potential or the Pollmann-Büttner potential. The energy spectrum and the eigenfunctions are calculated for both cases. The results show that the Pollmann-Büttner model, using the corresponding parameters obtained from ab initio calculations, provides better agreement with the experimental results.

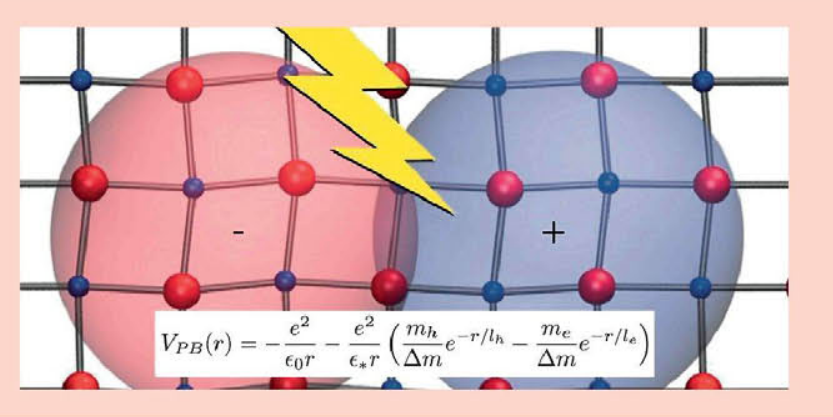

1 Introduction Metal-organic tri-halides perovskites are semiconductor materials that have revolutionized the research of thin film solar cells. With the first prototypes demonstrated six years ago [1], record cell efficiencies have surpassed the barrier of $20 \%[2,3]$. Methylammonium ( $\mathrm{MA}=\mathrm{CH}_{3} \mathrm{NH}_{3}^{+}$) lead iodide $\left(\mathrm{MAPbI}_{3}\right)$ is one of the most studied members of this family, and it has been applied as photon absorber and charge transporting material $[4,5]$. $\mathrm{MAPbI}_{3}$ has three crystal structures (orthorhombic, tetragonal and cubic, in order of increasing temperature) [6-9]. The three phases differ by small changes of the lattice vectors, rotations of the characteristic $\mathrm{PbI}_{6}$ octahedra, and the orientation of the $\mathrm{CH}_{3} \mathrm{NH}_{3}^{+}$cations, which is subject to dynamic disorder in the tetragonal and cubic phases [10].

The electronic band structure of $\mathrm{MAPbI}_{3}$ has been explained on the basis of generalized density functional theory (hybrid functionals) or Green functions GW calculations, in both cases including the spin-orbit coupling [1113]. For the orthorhombic phase, the valence band maximum (VBM) and the conduction band minimum (CBM) are located at the $\Gamma$-point corresponding to the 48 -atoms unit cell, and the fundamental gap is $1.68 \mathrm{eV}$ [14]. Both the VBM and CBM are doubly degenerated, with nearly symmetric effective mass tensors.

Exciton peaks are observed in the light absorption spectra at low temperature [15-17], just below the interband absorption edge, or melded with it, depending on the temperature. According to the Wannier-Mott model [18, 19], the exciton is similar to a hydrogen atom with the proton and electron masses replaced by the hole and electron effective masses, and the Coulomb interaction is screened by a dielectric constant $\varepsilon$. Therefore, the exciton binding energy and the Bohr radius are $R y=\mu e^{4} / 2 \hbar^{2} \varepsilon^{2}$ and $a_{\mathrm{ex}}=\hbar^{2} \varepsilon / \mu e^{2}$, where $\mu=m_{\mathrm{e}} m_{\mathrm{h}} /\left(m_{\mathrm{e}}+m_{\mathrm{h}}\right)$ is the reduced electron-hole mass.

One distinct feature of $\mathrm{MAPbI}_{3}$ is the large difference between the static dielectric constant $\varepsilon_{0}$ and the high frequency (ion-clamped) constant $\varepsilon_{\infty}$, i.e., for frequencies higher than those of the phonon absorption. Values of $\varepsilon_{\infty}$ in the range 4.5-6.5 have been calculated [11-13, 20], while values close to 25 have been estimated for $\varepsilon_{0}[12,20]$. Such difference is larger than in traditional inorganic semiconductor and should cause important polaron effects, such as 
Table 1 Parameters defining the polarons in $\mathrm{MAPbI}_{3}$.

\begin{tabular}{lll}
\hline Dielectric constants & $\varepsilon_{\infty}$ & $\varepsilon_{\infty} / \varepsilon_{0}$ \\
& $5.32^{1}$ & $0.236^{2}$ \\
LO phonon energy & $E_{\mathrm{LO}}$ & \\
& $38.5 \mathrm{meV}^{3}$ & \\
Coupling constants & $\alpha_{\mathrm{e}}$ & $\alpha_{\mathrm{h}}$ \\
& 1.18 & 1.28 \\
Bare carrier masses & $m_{\mathrm{e}} / m_{0}$ & $m_{\mathrm{h}} / m_{0}$ \\
& $0.190^{1}$ & $0.225^{1}$ \\
Polaron masses & $m_{\mathrm{e}}^{*} / m_{0}$ & $m_{\mathrm{h}}{ }^{*} / m_{0}$ \\
& 0.228 & 0.273 \\
Polaron radii & $l_{\mathrm{e}}$ & $l_{\mathrm{h}}$ \\
& $22.8 \AA$ & $21.0 \AA$ \\
Polaron shift & $\Delta E_{p}^{\mathrm{e}}$ & $\Delta E_{p}^{\mathrm{h}}$ \\
& $-45.3 \mathrm{meV}^{1}$ & $-49.3 \mathrm{meV}^{1}$ \\
\hline
\end{tabular}

${ }^{1}$ Ref. [13]. ${ }^{2}$ Ref. [20]. ${ }^{3}$ Ref. [30].

the effective mass and gap renormalization, as well as nonhydrogenic exciton states. For the latter, immediately arises the question whether the screening constant $\varepsilon$ should be the static dielectric constant $\varepsilon_{0}$ or the high frequency $\varepsilon_{\infty}$. Using the values listed in Table 1 , the static and the high frequency dielectric constants lead to very different values of the exciton binding energy $R y_{0}=2.8 \mathrm{meV}$ and $R y_{\infty}=50 \mathrm{meV}$, respectively. Such different energies lead to different conclusions with respect to exciton dissociation due to thermal excitation, as well as to different interpretation of luminescence and transport properties.

In fact, the difference between $\varepsilon_{0}$ and $\varepsilon_{\infty}$ expresses the electric polarization associated to the optical phonons and the electron-phonon interaction. The stationary states are coupled states of electronic and the vibrational phonon field. The quantum calculation of these coupled states is beyond the current capabilities of ab initio methods. Model Hamiltonians $[21-23]$ allow one to map the coupled electron-phonon excitations into effective electronic states. and to obtain the energies of stationary states. Even when simplifying approximations are inherent in the models, they can provide a criterion on the relevant dielectric screening constants. In this Letter, we apply the model Hamiltonians of Haken [21, 22] and that of Pollmann and Büttner [23] to the exciton spectrum. This formalism is applicable only to the low temperature orthorhombic phase because in the tetragonal phase the static dielectric constant increases strongly, associated to dynamical reorientation of $\mathrm{CH}_{3} \mathrm{NH}_{3}^{+}$cations, and the exciton effects practically disappear [24-28]. This formalism cannot be applied to permanent rotating dipoles. Also, the orientation dynamics is much slower than the ionic polarization and its effect upon the excitons may depend on the exciton lifetime [29].

2 Theoretical models The strengths of the electronoptical phonon and hole-optical phonon interactions are given by the coupling constants

$$
\alpha_{\mathrm{e}, \mathrm{h}}=\sqrt{m_{\mathrm{e}, \mathrm{h}} e^{4} / 2 \hbar^{2} \varepsilon_{*}^{2} E_{\mathrm{LO}}},
$$

where $E_{\mathrm{LO}}$ is the energy of the longitudinal optical phonon. This model was developed for simple crystals that display one single LO phonon branch. For this application, we have chosen $E_{\mathrm{LO}}$ as the shift of the main peak in the $\mathrm{MAPbI}_{3}$ Raman spectrum [30]. The ionic screening parameter appearing in Eq. (1) is $1 / \varepsilon_{*}=1 / \varepsilon_{\infty}-1 / \varepsilon_{0}$.

For transport properties, relevant after exciton dissociation, polaron masses must be considered rather than the bare electronic masses computed with fixed ions. They can be estimated using the Fröhlich's continuum theory of the large polaron [31], which predicts

$$
m_{\mathrm{e}, \mathrm{h}}^{*}=m_{\mathrm{e}, \mathrm{h}}\left(1+\frac{\alpha_{\mathrm{e}, \mathrm{h}}}{6}\right) .
$$

The polaron bands undergo an additional shift given by $\Delta E_{p}^{\mathrm{e}, \mathrm{h}}=-\alpha_{\mathrm{e}, \mathrm{h}} E_{\mathrm{LO}}$. With the data of Table 1, this leads to a reduction of the electronic band gap by $95 \mathrm{meV}$.

The Haken $(\mathrm{H})$ model $[21,22]$ describes two interacting polarons, each one with a radius much smaller than the exciton effective radius, and expresses the effective potential for the electron-hole interaction as

$$
V_{\mathrm{H}}(r)=-\frac{e^{2}}{\varepsilon_{0} r}-\frac{e^{2}}{2 \varepsilon_{*} r}\left(\mathrm{e}^{-r / l_{0}}+\mathrm{e}^{-r / h}\right) .
$$

Here $l_{\mathrm{e}, \mathrm{h}}=\sqrt{\hbar^{2} / 2 m_{\mathrm{e}, \mathrm{h}} E_{\mathrm{LO}}}$ are the electron- and holepolaron radii determined using bare band electron and hole effective masses. The polaron effective mass parameters must be used in the kinetic energy terms of the Hamiltonian [32].

A refined model, proposed by Pollmann and Büttner [23] (PB), expresses the electron-hole interaction potential as

$$
V_{\mathrm{PB}}(r)=-\frac{e^{2}}{\varepsilon_{0} r}-\frac{e^{2}}{\varepsilon_{* r}}\left(\frac{m_{\mathrm{h}}}{\Delta m} \mathrm{e}^{-r / h_{\mathrm{h}}}-\frac{m_{\mathrm{e}}}{\Delta m} \mathrm{e}^{-r / l_{\mathrm{e}}}\right),
$$

with $\Delta m=m_{\mathrm{h}}-m_{\mathrm{e}}$. This potential was derived assuming that the polaron lengths $l_{\mathrm{e}, \mathrm{h}}$ are much smaller than the effective exciton radius, which entered as a variational parameter in the original calculations [23]. The bare band electron and hole masses must be used in the kinetic energy terms of the PB Hamiltonian.

In the present work, the exciton energies are obtained solving numerically the radial Schrödinger equation for the relative coordinate wave function of the exciton $\Phi(r)$

$$
\frac{\mathrm{d}^{2} \Phi}{\mathrm{d} r^{2}}+\frac{2}{r} \frac{\mathrm{d} \Phi}{\mathrm{d} r}+\left(\frac{2 \mu}{\hbar^{2}}(E-V(r))-\frac{l(l+1)}{r^{2}}\right) \Phi=0,
$$

where $V(r)$ is the electron-hole interaction potential (Coulomb, $\mathrm{H}$, or PB), $l$ is the azimuthal quantum number, of which we only consider $l=0$ that are the optically active states. The Eq. (4) for $l=0$ has been solved integrating the equation starting from $r=0$ with the conditions $\Phi(0)>0$, $\Phi^{\prime}(0)=0$ and imposing $\Phi\left(r_{\mathrm{c}}\right)=0$, where $r_{\mathrm{c}}$ is a cutoff radius sufficiently large to mimic the boundary conditions at 
infinity. We have used exciton atomic units $a_{0}$ and $R y_{0}$ for the radius and energy, respectively. The cutoff radii $r_{c}$ are established solving the equation for the Coulomb potentials and comparing the numerical energies with the known exact solutions, i.e. $-1 / n^{2}$. The radial wave functions functions are normalized to unity.

The optical oscillator strengths are defined as

$$
f_{n}=\frac{2 m_{0}}{\hbar \omega_{n, 0}}\left|\left\langle\Psi_{n}^{\prime}|\boldsymbol{\xi} \cdot \hat{\boldsymbol{v}}| 0\right\rangle\right|^{2},
$$

where $m_{0}$ is the free electron mass, $h \omega_{n, 0}=E_{\mathrm{g}}^{*}+E_{n}$ is the transition energy, $|0\rangle$ and $\left|\Psi_{n}^{\prime}\right\rangle$ are the ground and excited states of the crystal, respectively, and $\hat{\boldsymbol{v}}=i[\hat{H}, \boldsymbol{r}] / \hbar$ is the velocity operator [33]. $E_{\mathrm{g}}^{*}$ is the renormalized gap (with the polaron shift), and $E_{n}$ are the eigenvalues of Eq. (4). We shall approximate $f_{n}$ by the expression for pure excitons, i.e., neglecting the phonon coupling. We obtain the simplified expression (see the Supporting Information)

$$
f_{n}=\frac{4 U_{c v}}{\hbar \omega_{n, 0}} \frac{\Omega_{\text {f.u. }}}{a_{0}^{3}}\left|\Phi_{n}(0)\right|^{2} .
$$

In the above expression, $\Omega_{\text {f.u. }}$ is the normalization volume of the center-of-mass part of the exciton envelope wave function, which we consider as the volume of one formula unit, i.e., one fourth of the unit cell volume $952.5 \AA^{3}$. With this convention, the oscillator strength is equivalent to the values reported elsewhere $[14,16]$. Using first principles calculations (see the Supporting Information) we have calculated the parameter

$$
U_{c v}=\frac{m_{0}}{2} \sum_{c v} \sum_{\alpha=x, y, z} \frac{\left|\left\langle u_{c 0}\left|\hat{v}_{\alpha}\right| u_{v 0}\right\rangle\right|^{2}}{3}=1.706 \mathrm{eV}
$$

The factor $1 / 3$ and the sum in $\alpha$ correspond to isotropic average of the crystal orientations. $u_{v 0}$ and $u_{c 0}$ are the Bloch functions of the valence band maximum and conduction band minimum, which in this case are both doubly degenerate.

3 Results and discussion Both the $\mathrm{H}$ and $\mathrm{PB}$ potentials behave like a Coulomb potential for very large distance $\left(r \gg l_{\mathrm{e}}, l_{\mathrm{h}}\right)$ or very short distances $\left(r \ll l_{\mathrm{e}}, l_{\mathrm{h}}\right)$, screened by the low and high frequencies dielectric constants, respectively. Figure 1 shows in logarithmic scale, the limiting Coulomb potentials screened by $\varepsilon_{0}$ and $\varepsilon_{\infty}$. These are represented by the straight lines, enclosing the $\mathrm{H}$ and PB potentials, that interpolate the limiting cases. Horizontal lines represent the eigenenergies of the exciton relative motion. The axes in the figure are in units of static (fully screened) exciton radius and $a_{0}=\hbar^{2} \varepsilon_{0} / \mu e^{2}$ and exciton energy $R y_{0}=\mu e^{4} / 2 \hbar^{2} \varepsilon_{0}^{2}$. In these units, the static Coulomb potential is given by $-2 / r$ and the exciton eigenenergies are $E_{n}^{0}=-1 / n^{2}$. The Coulomb potential and the hydrogenic energies defined by $\varepsilon_{\infty}$ are $E_{n}^{\infty}=-\varepsilon_{r}^{2} / n^{2}$, where $\varepsilon_{r}=\varepsilon_{0} / \varepsilon_{\infty}$. For the parameters of $\mathrm{MAPbI}_{3}\left(\varepsilon_{r}=4.24\right)$, the

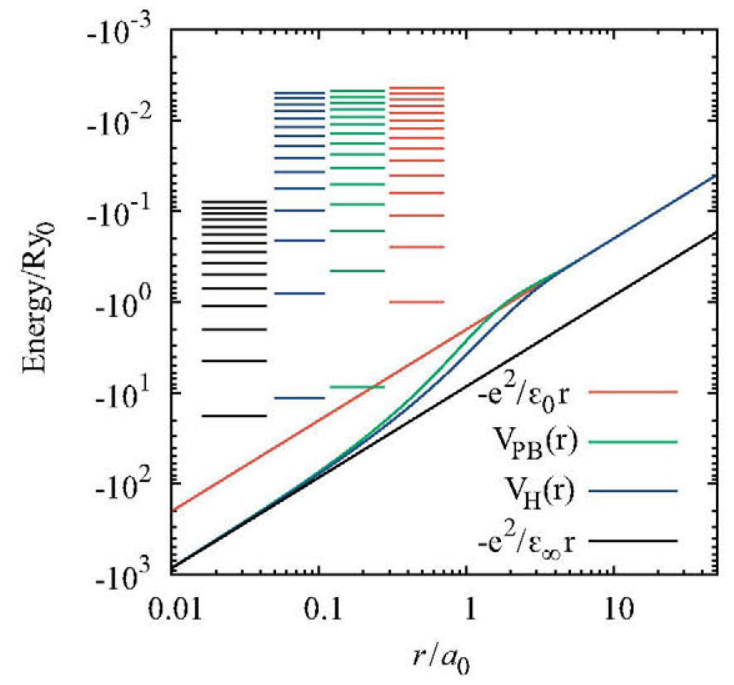

Figure $1 \mathrm{H}$ and $\mathrm{PB}$ potentials compared with the Coulomb potential screened by $\varepsilon_{0}$ and $\varepsilon_{\infty}$. Also shown are the eigenenergies for each potential.

lowest exciton levels are $E_{n}^{\mathrm{H}}=-11.26$ and $E_{n}^{\mathrm{PB}}=-8.65$ for the $\mathrm{H}$ and $\mathrm{PB}$ potentials (see Table 1 in Supporting Information). These values represent a significant correction to either $E_{1}^{0}=-1$ or $E_{1}^{\infty}=-18$. The excited exciton energies of $\mathrm{H}$ and $\mathrm{PB}$ potentials approach the values $-1 / n^{2}$ for high $n$.

In order to compare the energies $E_{1}^{\mathrm{H}}$ and $E_{1}^{\mathrm{PB}}$ one must consider that the reduced mass $\mu$ in $R y_{0}$ is defined either by the polaron reduced masses or the bare reduced masses in the first and second model, respectively. In absolute units, $E_{1}^{\mathrm{H}}=-37 \mathrm{meV}$ and $E_{1}^{\mathrm{PB}}=-24 \mathrm{meV}$. Is seems that the $\mathrm{PB}$ value is in better agreement with the experimental values near $19 \mathrm{meV}[28,34]$.

According to Eq. (6), the oscillator strengths depend on the potential model only through $\Phi_{n}(0)$. Figure 2 shows the values of $\pi n^{3}\left|\Phi_{n}(0)\right|^{2}$ (see also Table 1 in Supporting Information). For the $\varepsilon_{0}$-hydrogenic potential, $\left|\Phi_{n}(0)\right|^{2}=1 / \pi n^{3}$. Therefore, $\pi n^{3}\left|\Phi_{n}(0)\right|^{2}$ is the oscillator strength relative to the hydrogenic one. For the $\varepsilon_{\infty}$-hydrogenic potential, $\pi n^{3}\left|\Phi_{n}(0)\right|^{2}=\varepsilon_{r}^{3}$. The $n=1$ state approaches the later limit. For higher levels, the oscillator strengths become proportional to the hydrogenic oscillator strengths. The proportionality constant is fitted to $\beta_{\mathrm{H}}=4.282$ and $\beta_{\mathrm{PB}}=4.214$ in each case. The fitting function was $f(n)=\beta+\gamma /\left(n-n_{0}\right)$, and the fitted $\beta$ are stable for any subset of data with $n>7$. Let us note that

$$
\beta \simeq \frac{\varepsilon_{0}}{\varepsilon_{\infty}}
$$

The $n=1$ oscillator strength, according to Eq. (6), is 0.013 (PB model). This is close to the value 0.02 reported by Ishihara [14]. This parameter provides another argument against the $\varepsilon_{0}$-hydrogenic model, for which an oscillator strength $\sim 64$ times smaller is predicted. 


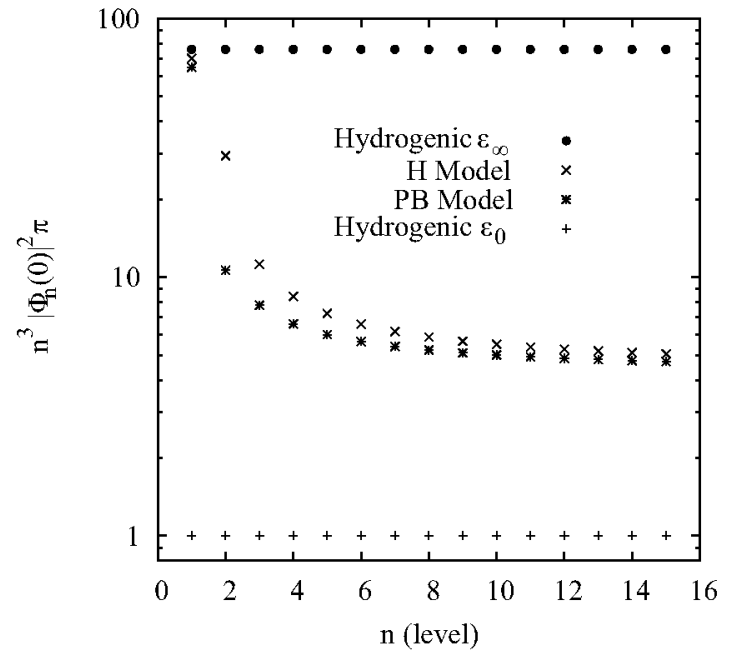

Figure 2 Oscillator strengths of the $\varepsilon_{\infty}$-hydrogenic, Haken $(\mathrm{H})$ and Pollman-Büttner (PB) excitons relative to the strengths of hydrogenic exciton determined by $\varepsilon_{0}$.

The second exciton level is only $2.7 \mathrm{meV}(\mathrm{H})$ or $1.3 \mathrm{meV}$ (PB) below the edge of the continuum spectrum, and their oscillator strengths are one order of magnitude smaller than for the main line $(n=1)$, making these transitions practically undetectable in the optical spectra. Higher energies approach to the sequence $E_{\mathrm{g}}^{*}-R y_{0} / n^{2}$, as can be seen in Table 1 of Supporting Information. This result, together with the approximation of the energies by the sequence $E_{\mathrm{g}}^{*}-R y_{0} / n^{2}$, leads to to a constant absorption spectrum near the band gap energy similar to the case of hydrogenic exciton $[35,36]$,

$$
\alpha\left(E_{\mathrm{g}}^{*}\right)=\frac{4 \pi e^{2} \hbar U_{c v}}{E_{\mathrm{g}}^{*} n_{r} c m_{0}} \frac{\beta}{a_{0}^{3} R y_{0}},
$$

where $n_{r}$ is the refraction index and can be approximated by $\sqrt{\varepsilon_{\infty}}$. Using the material parameters of $\mathrm{MAPbI}_{3}$, $\alpha\left(E_{\mathrm{g}}\right) \sim 3.1 \times 10^{4} \mathrm{~cm}^{-1}$. This value is enhanced with respect to the hydrogenic model by a factor $\varepsilon_{0} / \varepsilon_{\infty}$.

To interpret the absorption experiments and to determine the band gap, one needs to know whether the onset of the continuous absorption spectrum corresponds to the exciton continuum spectrum, or to the accumulation of discrete lines below the band gap. In other words, what is the energy range of constant absorption coefficient given by Eq. (9). Considering that the higher exciton levels are within $2.7 \mathrm{meV}$ of the continuum, and that the exciton absorption spectrum is dominated by the fundamental state, one can conclude that the band gap coincides with the absorption threshold after filtering the first exciton peak. On the other hand, the polaronic effect downshifts the gap by $95 \mathrm{meV}$. Therefore, the measured gap $1.68 \mathrm{eV}$ should be understood as an electronic gap of $1.78 \mathrm{eV}$ decreased by the polaron shift

Several kinds of estimations of the exciton binding energy in $\mathrm{MAPbI}_{3}$ have been reported. The first method was employed by Hirasawa et al. [15], and repeated later with improved accuracy by Tanaka et al. [16]. They measured the exciton diamagnetic coefficient in magnetoabsorption spectra, and related the measurements with the binding energy in the framework of the hydrogenic model with the high frequency dielectric constant. With this model, Tanaka et al. determined a binding energy of $50 \mathrm{meV}$. The use of the high frequency dielectric constants was a choice of the model, and not determined by the experiments.

The second method has been applied by Sun et al. [34]. They obtained the binding energy $(19 \mathrm{meV}$ ) by fitting the photoluminescence intensity as a function of temperature with an Arrhenius equation, not using any model of the exciton states. Huang and Lambrecht [37] have argued, in a study of cesium tin halide perovskites, that the photoluminescence temperature dependence just gives information on the free exciton linewidth or the binding energies of bound excitons, but not on free excitons. However, Even et al. [27] fitted the absorption spectrum using the Wannier-Mott exciton model and obtained a similar value for the binding energy, and reported an effective dielectric constant $\varepsilon_{\text {eff }}=11$. This value of the dielectric constants, together with the assumed reduced mass $0.16 m_{0}$ [27] means a binding energy of $19 \mathrm{meV}$. Another method independent of the dielectric function has been used by Miyata et al. [28], who performed magneto-absorption experiments with very high magnetic fields, determining a value of $16 \pm 2 \mathrm{meV}$. It is interesting that Miyata et al. were able to detect the $2 \mathrm{~s}$ exciton state for high magnetic field and extrapolated a $1 \mathrm{~s}-2 \mathrm{~s}$ difference of $15 \mathrm{meV}$ at low magnetic field. Henceforth, assuming the hydrogenic model, they estimated the binding energy in $20 \mathrm{meV}$. However, extrapolating the Landau levels of the free exciton spectrum they obtained the precise value of $16 \mathrm{meV}$. This observation agrees with our result that the $2 \mathrm{~s}$ state is within $2.7 \mathrm{meV}$ of the free exciton edge.

We wish to stress that we have not fitted any parameter in this work, which would bring the exciton binding energies in closer agreement with the recent experimental results. The parameters with larger uncertainty are the dielectric constants and the LO phonon energy. The only available experimental value of $\varepsilon_{\infty}=6.5$ [15] is larger than the $a b$ initio value used here and that value would reduce the calculated binding energy. The measurement of $\varepsilon_{\infty}$ is rather old, with few published details, and a new determination for present-day thin films would be welcomed. The LO phonon energy $E_{\mathrm{LO}}$ has been chosen from the more prominent peak in the calculated Raman spectrum of $\mathrm{MAPbI}_{3}$ [30], which is close to LO phonon energies in II-VI and III-V semiconductors. As mentioned above, the model Hamiltonians were developed assuming a unique LO phonon energy. The Raman spectrum of $\mathrm{MAPbI}_{3}$ shows bands at lower wave numbers. Using and average energy of the Raman active peaks, which do not have necessarily LO character, may lead to lower exciton binding energy. An extension of the PB Hamiltonian to include 
several LO phonon branches would be a better founded approach.

In summary, we have calculated the exciton binding energies and oscillator strengths using two model Hamiltonians of the exciton-phonon coupled system. The Pollmann-Büttner model Hamiltonian gives a binding energy in good agreement with recent experimental determinations. The calculated oscillator strength of the main exciton line agrees with the value estimated from experiments, while the strengths of higher transitions are much smaller.

Supporting Information Additional supporting information may be found in the online version of this article at the publisher's website.

Acknowledgements We acknowledge computer time from the Jülich Supercomputing Centre (JSC) under the MOHPSOPHIA project, and from the Madrid Supercomputing and Visualization Center (CeSViMa). We acknowledge support from FONDECYT Grant. No. 1150538 and project BOOSTER (ENE2013-46624-C4-2-R). We thank J. C. Conesa, P. Palacios, and $\mathrm{C}$. Trallero-Giner for interesting discussions that motivated this work.

\section{References}

[1] A. Kojima, K. Teshima, Y. Shirai, and T. Miyasaka, J. Am. Chem. Soc. 131, 6050-6051 (2009).

[2] 2015, NREL chart on record cell efficiencies

[3] M. Jacoby, Chem. Eng. News 92, 21 (2014).

[4] L. Etgar, P. Gao, Z. Xue, Q. Peng, A. K. Chandiran, B. Liu, M. K. Nazeeruddin, and M. Grätzel, J. Am. Chem. Soc. 134(42), 17396-17399 (2012).

[5] N. G. Park, J. Phys. Chem. Lett. 4(15), 2423-2429 (2013).

[6] O. Knop, R. E. Wasylishen, M. A. White, T. S. Cameron, and M. J. M. van Ooort, Can. J. Chem. 68, 412-422 (1990).

[7] T. Baikie, Y. Fang, J. M. Kadro, M. Schreyer, F. Wei, S. G. Mhaisalkar, M. Graetzel, and T. J. White, J. Mater. Chem. A 1, 5628-5641 (2013).

[8] C. C. Stoumpos, C. D. Malliakas, and M. G. Kanatzidis, Inorg. Chem. 52, 9019-9038 (2013).

[9] Y. Kawamura, H. Mashiyama, and K. Hasebe, J. Phys. Soc. Jpn. 71, 1694-1697 (2002).

[10] R. E. Wasylishen, O. Knopp, and J. B. Macdonald, Solid State Commun. 56, 581-582 (1985)

[11] P. Umari, E. Mosconi, and F. De Angelis, Sci. Rep. 4, 4467 (2014).

[12] F. Brivio, K. T. Butler, A. Walsh, and M. van Schilfgaarde, Phys. Rev. B 89, 155204 (2014).
[13] E. Menéndez-Proupin, P. Palacios, P. Wahnón, and J. C. Conesa, Phys. Rev. B 90, 045207 (2014).

[14] T. Ishihara, J. Lumin. 60-61, 269-274 (1994).

[15] M. Hirasawa, T. Ishihara, T. Goto, K. Uchida, and N. Miura, Physica B 201, 427-430 (1994).

[16] K. Tanaka, T. Takahashi, T. Ban, T. Kondo, K. Uchida, and N. Miura, Solid State Commun. 127, 619-623 (2003)

[17] V. D'Innocenzo, G. Grancini, M. J. P. Alcocer, A. R. S Kandada, S. D. Stranks, M. M. Lee, G. Lanzani, H. J. Snaith, and A. Petrozza, Nature Commun. 5, 3586 (2014).

[18] G. Wannier, Phys. Rev. 52, 191-197 (1937).

[19] R. Knox, Theory of excitons, Solid State Physics: Supplement 5 (Academic Press, 1963).

[20] F. Brivio, A. B. Walker, and A. Walsh, APL Materials 1, 042111 (2013).

[21] H. Haken, Z. Phys. 146, 527-554 (1956).

[22] H. Haken, Fortschr. Phys. 6, 271-334 (1958).

[23] J. Pollmann and H. Büttner, Phys. Rev. B 16, 4480-4490 (1977).

[24] N. Onoda-Yamamuro, T. Matsuo, and H. Suga, J. Phys. Chem. Solids 53, 935-939 (1992).

[25] Q. Lin, A. Armin, R. C. R. Nagiri, P. L. Burn, and P. Meredith, Nature Photon. 9, 106-112 (2015).

[26] J. M. Frost, K. T. Butler, and A. Walsh, APL Materials 2(8), 081506 (2014).

[27] J. Even, L. Pedesseau, and C. Katan, J. Phys. Chem. C 118 , $11566-11572$ (2014).

[28] A. Miyata, A. Mitiouglu, P. Plochocka, O. Portugall, J. T. W. Wang, S. D. Stranks, H. J. Snaith, and R. J. Nicholas, Nature Phys. 11, 582-587 (2015).

[29] S. T. A. G. Melissen, F. Labat, P. Sautet, and T. Le Bahers, Phys. Chem. Chem. Phys. 17, 2199-2209 (2015).

[30] C. Quarti, G. Grancini, E. Mosconi, P. Bruno, J. M. Ball, M. M. Lee, H. J. Snaith, A. Petrozza, and F. De Angelis, J. Phys. Chem. Lett. 5, 279-284 (2014).

[31] J. T. Devreese and A. S. Alexandrov, Rep. Prog. Phys. 72, 066501 (2009).

[32] H. Haken, J. Phys. Radium 17, 826-828 (1956).

[33] R. del Sole and R. Girlanda, Phys. Rev. B 48, 11789-11795 (1993).

[34] S. Sun, T. Salim, N. Mathews, M. Duchamp, C. Boothroyd, G. Xing, T. C. Sumbce, and Y. M. Lam, Energy Environ. Sci. 7, 399-407 (2014).

[35] G. Grosso and G. Pastori-Parravicini, Solid State Physics, 1 st edition (Academic Press, San Diego, 2000).

[36] R. J. Elliot, Phys. Rev. 108, 1384-1389 (1957).

[37] L. Y. Huang and W. R. L. Lambrecht, Phys. Rev. B 88, 165203 (2013). 